\title{
Editorial
}

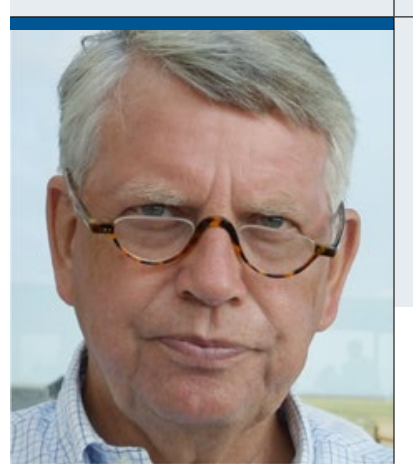

„Der Nutzen niedriger LDL-C-Konzentrationen steht den vielen

Patienten mit erhöhten kardiovaskulären Risiken gegenüber, bei

denen sich eine solche Cholesterinsenkung mit ausschließlich

diätetischen Maßnahmen nicht realisieren lässt."

Prof. Dr. Gerald Klose

Praxis Dres. Beckenbauer \& Maierhof

Am Markt 11, 28195 Bremen

\section{„What should you eat to live a heart healthy life?"}

$\mathrm{K}$ örperliche Aktivität und gute Ernährung sind Komponenten eines gesunden Lebensstils, der in Leitlinien zur kardiovaskulären Prävention als Voraussetzung einer Risikofaktorkontrolle aufgeführt ist. Konkrete Empfehlungen basierten bis vor Kurzem meist auf Erkenntnissen aus Beobachtungsstudien. Aktuelle Metaanalysen gaben nun Anlass, die Evidenz für über Jahrzehnte getroffene Aussagen zum Zusammenhang eines bestimmten Nahrungsfett-Konsums mit einer kardiovaskulären Risikoerhöhung zu hinterfragen. Mit fehlender Evidenz aus randomisierten kontrollierten Studien trugen diese Diskussionen zur Änderung der US Dietary Guidelines 2015 bei; bei einer Sitzung des ESCKongresses 2015 in London mit dem Titel „What should you eat to live a heart healthy life?" wurden diese als "seismisch" apostrophiert. Aufgehoben wurde etwa die generelle Begrenzung der Aufnahme von Nahrungscholesterin (z. B. Eier) und Fetten.

Als Konzept des Diogenes-Trial handelte Prof. Thomas Meinert Larsen, Kopenhagen, in dieser Sitzung die verbesserte Gewichtsregulation unter eiweißreicher Kost mit niedrigem glykämischem Index ab. Dr. Simon Poole, Cambridge, berücksichtigte die Perspektiven, die sich aus der Umsetzung einer mediterranen Ernährung entsprechend der PREDIMED-Studie ergeben. Auf die methodischen Aspekte von Ernährungsstudien, die der Komplexität der Zusammenhänge gerecht werden müssen, verwies PD Dr. Neil Thomas, Birmingham. Die Kernbotschaft von Dr. Steen Stender, Hellerup, lautete, den Verzicht auf Trans-Fettsäuren möglichst umzusetzen. Sein Apell basierte auf der Erkenntnis, dass die tägliche Aufnahme von $5 \mathrm{~g}$ Trans-Fettsäuren mit einer 23\%igen kardiovaskulären Risikoerhöhung verbunden ist. Nach dieser Sitzung schrieb Melissa Walton-Shirley, Medscape International 2015, einen Kommentar mit der Überschrift „Imagine Our World if We Taught It How to Eat": It was probably the most important series of the entire ESC 2015 meeting because they advocated for nutrients whose side effects are nil (for most) and are readily available for mass consumption (in most places).

Die Themenauswahl dieses CardioVasc-Schwerpunktes Lipide scheint diesen Kommentar zu bestätigen. Christine Dawczynski und Stefan Lorkowski vom Institut für Ernährungswissenschaften, Friedrich-Schiller-Universität Jena, referieren detailliert und auf dem neuesten Stand ernährungsphysiologische und epidemiologische Daten zur Fettsäurezusammensetzung in der Ernährung. Zu deren Stellenwert in der Prävention kann sich der Leser selbst ein Bild machen. Als Fazit nennen die Autoren die Notwendigkeit, die mit der Nahrung aufgenommenen Fette entsprechend zu modifizieren, und propagieren einen möglichst frühen Beginn dieser Maßnahme. Birgit-Christiane Zyriax, Präventive Medizin, Universitäres Herzzentrum Hamburg GmbH (UHZ) des UKE, referiert über die Outcome-Evidenz der PREDIMED-Studie und nimmt Stellung zur Frage, ob deren Ergebnisse auch auf andere Bevölkerungsgruppen übertragbar sind.

Der bewiesene präventive Nutzen niedriger LDLCholesterin-Konzentrationen steht den vielen Patienten mit erhöhten kardiovaskulären Risiken gegenüber, bei denen sich eine solche LDL-Senkung mit ausschließlich diätetischen Maßnahmen nicht realisieren lässt. Die EAS/ESC-Leitlinie zur Lipidtherapie enthält Hinweise, wie sich das Plasmalipidprofil durch Lebensstiländerung verbessern lässt. Erwähnt wird etwa das „Functional Food“, angereichert mit Phytosterolen. Eberhard Windler, Präventive Medizin, UHZ, und Birgit-Christiane Zyriax diskutieren umfassend darüber, ob oder wann die Supplementierung von Phytosterinen eine Perspektive oder ein Risiko bedeuten kann.

Ihr

Prof. Dr. Gerald Klose 